\title{
Probing the neutrino reactor anomaly with the T2K near detector
}

\section{Davide Sgalaberna* for the T2K Collaboration}

ETH Zurich, Switzerland

E-mail: davide.sgalaberna@cern.ch

\begin{abstract}
Hints of possible electron neutrino disappearance that might be explained with the presence of additional sterile neutrinos $\left(v_{s}\right)$ have been observed in the so-called reactor and Gallium anomalies. This can be tested with the near detector (ND280) at the T2K experiment, a long baseline neutrino oscillation experiment designed to precisely measure the PMNS matrix by looking for the appearance of $v_{e}$ in a $v_{\mu}$ beam. Thanks to its short baseline ND280 can be used to study sterile neutrino oscillations. Furthermore a very good $v_{\mu}-v_{e}$ separation can be performed and the large part of $v_{\mu}$ background can be rejected. A full sensitivity study of $v_{e} \rightarrow v_{s}$ disappearance in the $3+1$ neutrino mixing model is presented and compared with the other experimental results.
\end{abstract}

The European Physical Society Conference on High Energy Physics

18-24 July, 2013

Stockholm, Sweden

\footnotetext{
* Speaker.
} 


\section{Sterile neutrinos}

Sterile neutrinos are right-handed and do not undergo weak interaction. Their existence can be studied through the mixing with the active neutrinos. The $3+1$ model is the minimal extension of the standard three neutrino mixing with the addition of one sterile neutrino field $v_{s}$ to the standard three active neutrino fields $\left(v_{e}, v_{\mu}\right.$ and $\left.v_{\tau}\right)$.

In this model the $v_{e}$ to $v_{s}$ mixing is described by the survival probability

$$
P_{v_{e} \rightarrow v_{e}}=1-\sin ^{2} 2 \theta_{e e} \cdot \sin ^{2}\left(\frac{1.27 \Delta m_{41}^{2} L_{V}}{E} \frac{[\mathrm{GeV}]}{[\mathrm{eV}]^{2}[\mathrm{~km}]}\right)
$$

where $L_{v}$ and $E$ are the neutrino flight path and energy and $\sin ^{2} 2 \theta_{e e}$ and $\Delta m_{41}^{2}$ are respectively the $v_{e} \rightarrow v_{s}$ transition amplitude and the mass square difference between the sterile and the standard neutrinos. Two indications of $v_{e} \rightarrow v_{s}$ disappearance come from the so-called "reactor neutrino anomaly" [2] and "Gallium anomaly" [3], [4], that can be explained assuming $\sin ^{2} 2 \theta_{e e} \sim 0.2$ 0.5 and $\Delta m_{41}^{2} \sim 1 \mathrm{eV}^{2} . \Delta m_{41}^{2}$ is much larger than the other two standard mass differences and is responsible for the short baseline oscillations.

\section{Sensitivity to sterile $v_{e}$ disappearance with the near detector at T2K}

The T2K (Tokai-to-Kamioka) experiment is a long baseline neutrino oscillation experiment that aims to precisely measure the PMNS matrix through the observation of $v_{\mu} \rightarrow v_{e}$ appearance. The neutrino beam is generated by a high intensity $31 \mathrm{GeV} / \mathrm{c}$ proton beam interacting on a carbon target and is detected by a near detector (ND280), $280 \mathrm{~m}$ from the target, and a far detector located $295 \mathrm{~km}$ away (Super Kamiokande). The neutrino beam is composed of about $99 \%$ of $v_{\mu}$ and the remaining $1 \%$ of $v_{e}$ [1]. Since ND280 is close to the neutrino production target, it can be sensitive to an indirect detection of non standard neutrino oscillations.

The $3+1$ model is used for the sensitivity study at ND280 to $v_{e} \rightarrow v_{s}$ where $U_{\mu 4}$ is assumed to be 0 in order to investigate the Gallium and reactor anomalies.

A clean $v_{e}$ sample with a purity of $67 \%$ is selected thanks to a very good $\mu$-e separation: the magnetic field allows the separation of opposite charged particles and thanks to the good performance of the TPC and using informations of the Electronic Calorimeter (ECal) the $99.8 \%$ of $\mu$ is rejected. Then binned templates are built using the $\mathrm{MC}$ reconstructed energy distribution in the CCQE hypothesis (figure 1).

The dependency on the oscillation parameters $\left(\sin ^{2} 2 \theta_{e e}, \Delta m_{41}^{2}\right)$ is introduced by weighting the $v_{e}$ component with the oscillation probability (equation 1.1). Neutrino flux, cross section, final state interaction and detector systematic uncertainties are taken into account introducing 50 nuisance parameters in the fit.

The MC sensitivity study at $90 \%$ C.L. is performed in the non oscillation hypothesis using a likelihood-ratio technique and the standard 2 d.o.f. confidence level method. The study is done with the data statistics until summer $2013\left(6 \times 10^{20}\right.$ p.o.t. $)$ taking into account all the systematic uncertainties and with all the statistics that $\mathrm{T} 2 \mathrm{~K}$ should collect during the next years $\left(8 \times 10^{21}\right.$ p.o.t.), considering only statistical uncertainties. As shown in figure 2, with the current statistics part of the "Gallium anomaly" can be investigated. In the future, considering also that flux systematic 


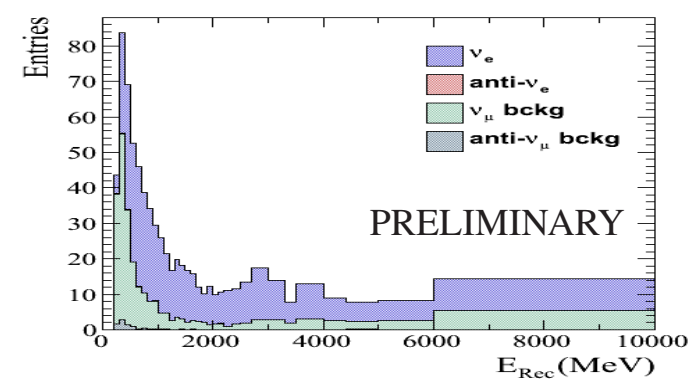

Figure 1: MC Reconstructed energy distribution at ND280 obtained after applying the $v_{e}$ selection. It corresponds to an exposure of $6 \times 10^{20}$ p.o.t.

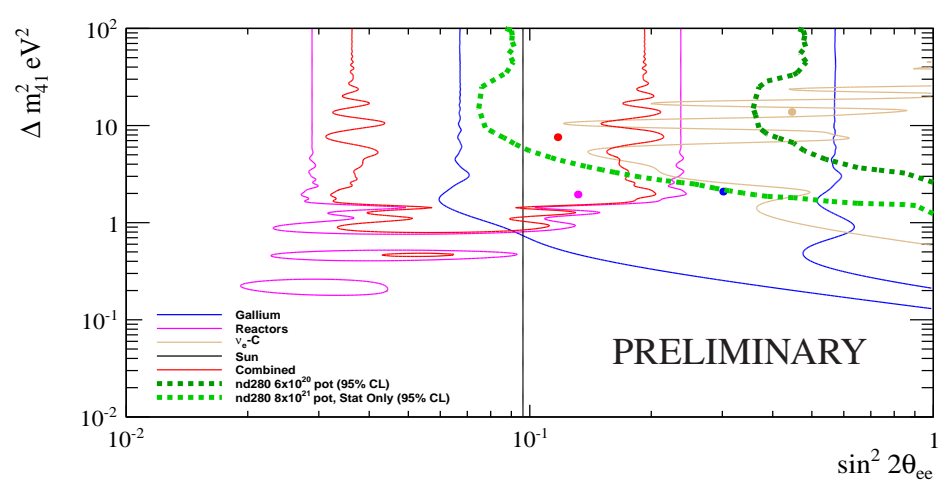

Figure 2: Comparison of the ND280 sensitivity with allowed region of $v_{e} \rightarrow v_{s}$ disappearance measured by other experiments [6]: Gallium experiments (blue), reactor anomaly (pink), solar experiments (black), $v_{e} C$ scattering data from LSND and KARMEN experiments (yellow) and the combined result (red). The dots correspond to the fitted minima of each different exclusion region. The ND280 sensitivity is shown taking into account all the systematic uncertainties with $6 \times 10^{20}$ p.o.t. and only statistical uncertainties with $8 \times 10^{21}$ p.o.t.

uncertainties will decrease thanks to the hadron production measurements of the NA61 experiment [5], also part of the allowed region of the reactor anomaly will be studied.

\section{References}

[1] K. Abe et al., Nucl. Instr. and Meth. A 659 (1) (2011) 106-135, arXiv:1106.1238v2

[2] T.Mueller,D.Lhuillier,M.Fallot,A.Letourneau,S.Cormon,etal.,Phys.Rev.C83,054615(2011)

[3] F. Kaether et al., Phys. Lett. B685, 47 (2010)

[4] J. N. Abdurashitov et al., Phys. Rev. C80, 015807 (2009), arXiv:0901.2200

[5] N. Abgrall et al. [NA61 Collaboration], arXiv:0908.1697 [nucl-ex]]

[6] C. Giunti, M. Laveder, Y. Li, Q. Liu, and H. Long, Phys.Rev. D86 (2012) 113014 